\title{
ON THE UMBILIC SET OF IMMERSED SURFACES IN THREE-DIMENSIONAL SPACE FORMS
}

\author{
GIOVANNI CATINO, ALBERTO RONCORONI, LUIGI VEZZONI
}

\begin{abstract}
We prove that under some assumptions on the mean curvature the set of umbilical points of an immersed surface in a 3-dimensional space form has positive measure. In case of an immersed sphere our result can be seen as a generalization of the celebrated Hopf theorem.
\end{abstract}

Key Words: immersed surfaces, Hopf theorem, mean curvature

AMS subject classification: 53C40, 53C42, 53A10

\section{INTRODUCTION}

In [12] Hopf proved his famous theorem

An immersed sphere of constant mean curvature in $\mathbb{R}^{3}$ is a round sphere.

The proof of Hopf's theorem involves the so-called Hopf differential Q, which is defined as the $(2,0)$-component of the second fundamental form of the surface (once regarded as a Riemann surface). The key point is that the zeros of $Q$ are the umbilical points of the surface and if the mean curvature is constant, $Q$ is holomorphic. Hence the set of umbilical points of a connected constant mean curvature surface can be only discrete or the whole surface. In the case of an immersed sphere Hopf showed that this set cannot be discrete and the theorem follows.

Hopf's theorem was generalized in three-dimensional space forms by Chern in [8] and, more recently, in 3-dimensional homogeneous spaces (see [13] and the references therein). The study of constant mean curvature spacial Riemannian manifolds is a central subject in differential geometry and there are many interesting results on this topic (see e.g. [1, 2, 3, 6, 7, 9, 14, 15, 18] and the reference therein).

Moreover, it is well known that the theorem cannot be generalized to surfaces of higher genus and the first counterexample was provided by Wente in [17] who constructed an immersed torus in $\mathbb{R}^{3}$ having constant mean curvature.

In order to state the result of the present paper we fix some notation for a given surface $M$ immersed in the 3-dimensional space form $\mathbb{F}^{3}(c)$ of curvature $c$ : $h$ is the second fundamental form; $H=\operatorname{tr} h$ is the mean curvature; $g$ is the induced metric on $M ; \stackrel{\circ}{h}=h-\frac{1}{2} \operatorname{tr}(h) g$ is the 
trace-free part of $h$. Moreover for $\epsilon>0$ we define the set

$$
\Omega_{\epsilon}=\{|\stackrel{\circ}{h}| \geq \epsilon\} .
$$

Notice that by definition $\Omega_{0}^{c}$ is the set of umbilical points of $M$.

The aim of this note is provide some sufficient conditions on the mean curvature which imply that the set of umbilical points $\Omega_{0}^{c}$ of an immersed surface in a 3-dimensional space form has positive measure. More precisely the result reads as follows

Theorem 1.1. Let $M$ be an immersed surface in $\mathbb{F}^{3}(c)$. Then, for every $0<\varepsilon \leq 1$, there exists a positive constant $C$, depending only on $\|H\|_{\infty}$ and $c$, such that

$$
C \operatorname{Vol}\left(\Omega_{\varepsilon}^{c}\right) \geq \frac{2}{\varepsilon^{4}} \int_{\Omega_{\varepsilon}^{c}}|\nabla \stackrel{\circ}{h}|^{2}|\stackrel{\circ}{h}|^{2}-\frac{1}{\varepsilon^{4}} \int_{\Omega_{\varepsilon}^{c}}|\nabla H|^{2}|\stackrel{\circ}{h}|^{2}+4 \pi \chi(M) .
$$

In particular, if

$$
\limsup _{\varepsilon \rightarrow 0} \frac{1}{\varepsilon^{4}} \int_{\Omega_{\varepsilon}^{c}}|\nabla H|^{2}|\stackrel{\circ}{h}|^{2}<\limsup _{\varepsilon \rightarrow 0} \frac{2}{\varepsilon^{4}} \int_{\Omega_{\varepsilon}^{c}}|\nabla \stackrel{\circ}{h}|^{2}|\stackrel{h}{h}|^{2}+4 \pi \chi(M),
$$

then $\operatorname{Vol}\left(\Omega_{0}^{c}\right)>0$.

As it will be clear from the proof, the result is sharp. That can be also deduced by checking that an ellipsoid of revolution (not spherical), which has two umbilical points, realizes the equality in (2) with $\chi(M)=2$.

As an immediate corollary we have

Corollary 1.2. Let $M$ be an immersed sphere in $\mathbb{F}^{3}(c)$. Then $\operatorname{Vol}\left(\Omega_{0}^{c}\right)>0$ if one of the following holds

1. $H$ is constant on $\Omega_{\varepsilon_{0}}^{c}$, for some $\varepsilon_{0}>0$;

2. $|\nabla H|^{2} \leq 2|\nabla h|^{2}$ on $\Omega_{\varepsilon_{0}}^{c}$, for some $\varepsilon_{0}>0$;

3. $\nabla H$ satisfies

$$
\limsup _{\varepsilon \rightarrow 0} \frac{1}{\varepsilon^{2}} \int_{\Omega_{\varepsilon}^{c}}|\nabla H|^{2}<8 \pi .
$$

Notice that the corollary in particular implies that if $H$ is constant, then the set of umbilical points on $M$ has positive measure which implies Hopf's theorem.

Notation. Throughout the paper the Einstein convention will be adopted and and the summation over repeated indices is omitted.

\section{Proof of Theorem 1.1 And Corollary 1.2}

As preliminary result we prove a Bochner-Weitzenböck type formula for the trace-free part of the second fundamental form of an immersed surface in a three dimensional space forms (for similar results we refer to $[4,5]$ ). 
Lemma 2.1. Let $M$ be an immersed surface in $\mathbb{F}^{3}(c)$. Then

$$
\frac{1}{2} \Delta|\stackrel{\circ}{h}|^{2}|\stackrel{\circ}{h}|^{2}=\left.2|\nabla| \stackrel{\circ}{h}\right|^{2}|\stackrel{\circ}{h}|^{2}-\stackrel{\circ}{h}_{i j} \nabla_{i}|\stackrel{\circ}{h}|^{2} \nabla_{j} H+\frac{1}{2}|\nabla H|^{2}|\stackrel{\circ}{h}|^{2}+R|\stackrel{\circ}{h}|^{4}+\stackrel{\circ}{h}_{i j} \nabla_{i} \nabla_{j} H|\stackrel{\circ}{h}|^{2},
$$

where $R$ is the the scalar curvature of $M$.

Proof. For any $p \in M$ we choose a local orthonormal frame $\left\{e_{1}, e_{2}, e_{3}\right\}$ in $\mathbb{F}^{3}(c)$ around $p$ such that $\left\{e_{1}, e_{2}\right\}$ are tangential to $M$. In this frame Codazzi and Gauss equations read as (see e.g. [11])

$$
\nabla_{k} h_{i j}-\nabla_{j} h_{i k}=0
$$

and the Riemannian curvature tensor of $M$ satisfies

$$
R_{i k j l}=c\left(g_{i j} g_{k l}+g_{i l} g_{j k}\right)+h_{i j} h_{k l}-h_{i l} h_{j k} .
$$

From (4) we get that $\stackrel{\circ}{h}=h-(H / 2) g$ satisfies the following equation

$$
\nabla_{k} \stackrel{\circ}{h}_{i j}-\nabla_{j} \stackrel{\circ}{h}_{i k}=\frac{1}{2}\left(\nabla_{j} H g_{i k}-\nabla_{k} H g_{i j}\right) \text {. }
$$

Taking the divergence of (6) we get

$$
\nabla_{k} \stackrel{\circ}{h}_{i j}=\frac{1}{2} \nabla_{j} H
$$

Moreover, taking the covariant derivative of (6) and tracing we obtain

$$
\Delta \stackrel{\circ}{h}_{i j}=\nabla_{j} \nabla_{k} \stackrel{\circ}{h}_{i k}+R \stackrel{\circ}{h}_{i j}+\frac{1}{2}\left(\nabla_{i} \nabla_{j} H-\Delta H g_{i j}\right) .
$$

From (7) and the commutation formula

$$
\nabla_{k} \nabla_{j} \stackrel{\circ}{h}_{i k}=\nabla_{j} \nabla_{k} \stackrel{\circ}{h}_{i k}+R \stackrel{\circ}{h}_{i j}
$$

we deduce

$$
\Delta \stackrel{\circ}{h}_{i j}=R \stackrel{\circ}{h}_{i j}+\nabla_{i} \nabla_{j} H-\frac{1}{2} \Delta H g_{i j} .
$$

From (9) we obtain

$$
\frac{1}{2} \Delta|\stackrel{\circ}{h}|^{2}=|\nabla \stackrel{\circ}{h}|^{2}+R|\stackrel{\circ}{h}|^{2}+\nabla_{i} \nabla_{j} H \stackrel{\circ}{h}_{i j}
$$

and then

$$
\frac{1}{2} \Delta|\stackrel{\circ}{h}|^{2}|\stackrel{\circ}{h}|^{2}=|\nabla \stackrel{\circ}{h}|^{2}|\stackrel{\circ}{h}|^{2}+R|\stackrel{\circ}{h}|^{4}+\nabla_{i} \nabla_{j} H \stackrel{\circ}{h}_{i j}|\stackrel{\circ}{h}|^{2} .
$$

The conclusion follows from the following formula:

$$
2|\stackrel{\circ}{h}|^{2}\left(|\nabla \stackrel{\circ}{h}|^{2}-\frac{1}{2}|\nabla H|^{2}\right)=\left.4|\nabla| \stackrel{h}{h}\right|^{2}|\stackrel{\circ}{h}|^{2}-2 \stackrel{\circ}{h}_{i j} \nabla_{i}|\stackrel{\circ}{h}|^{2} \nabla_{j} H
$$

which follows from [16, Formula 34] taking into account that $|\nabla h|^{2}=|\nabla h|^{2}+\frac{1}{2}|\nabla H|^{2}$; indeed by using (12) in (11) we get (3).

Now we can prove Theorem 1.1. 
Proof of Theorem 1.1. We consider the following positive function

$$
f_{\varepsilon}= \begin{cases}|\stackrel{\circ}{h}| & \text { in } \Omega_{\varepsilon} \\ \varepsilon & \text { in } \Omega_{\varepsilon}^{c},\end{cases}
$$

where, we recall that, $\Omega_{\varepsilon}=\{|\stackrel{\circ}{h}| \geq \varepsilon\}$. By multiplying (3) by $f_{\varepsilon}^{-4}$ and integrating over $M$ we get

$$
\begin{aligned}
0= & -\frac{1}{2} \int_{M} \Delta|\stackrel{\circ}{h}|^{2}|\stackrel{\circ}{h}|^{2} f_{\varepsilon}^{-4}+\left.2 \int_{M}|\nabla| \stackrel{\circ}{h}\right|^{2}|\stackrel{\circ}{h}|^{2} f_{\varepsilon}^{-4}-\int_{M} \stackrel{\circ}{h}_{i j} \nabla_{i}|\stackrel{\circ}{h}|^{2} \nabla_{j} H f_{\varepsilon}^{-4} \\
& +\frac{1}{2} \int_{M}|\nabla H|^{2}|\stackrel{\circ}{h}|^{2} f_{\varepsilon}^{-4}+\int_{M} R|\stackrel{h}{h}|^{4} f_{\varepsilon}^{-4}+\int_{M} \nabla_{i} \nabla_{j} H \stackrel{\circ}{h}_{i j}|\stackrel{\circ}{h}|^{2} f_{\varepsilon}^{-4} .
\end{aligned}
$$

Now we analyse the first, the second and the last integrals in (13). About the first one: by integrating by parts and taking into account that $\nabla f_{\varepsilon}=0$ in $\Omega_{\varepsilon}^{c}$ we get

$$
-\frac{1}{2} \int_{M} \Delta|\stackrel{\circ}{h}|^{2}|\stackrel{h}{h}|^{2} f_{\varepsilon}^{-4}=\left.\left.\frac{1}{2} \int_{M}|\nabla| \stackrel{\circ}{h}\right|^{2}\right|^{2} f_{\varepsilon}^{-4}-\left.4 \int_{\Omega_{\varepsilon}}|\nabla| \stackrel{\circ}{h}\right|^{2}|\stackrel{\circ}{h}|^{-2},
$$

i.e.

$$
-\frac{1}{2} \int_{M} \Delta|\stackrel{\circ}{h}|^{2}|\stackrel{\circ}{h}|^{2} f_{\varepsilon}^{-4}=\left.2 \int_{\Omega_{\varepsilon}}|\nabla| \stackrel{h}{h}\right|^{2}|\stackrel{\circ}{h}|^{2} f_{\varepsilon}^{-4}+2 \int_{\Omega_{\varepsilon}^{c}}|\nabla| \stackrel{h}{h}||^{2}|\stackrel{h}{h}|^{2} f_{\varepsilon}^{-4}-4 \int_{\Omega_{\varepsilon}}|\nabla| \stackrel{\circ}{h}||^{2}|\stackrel{\circ}{h}|^{-2},
$$

where we have used that $\left.\left.|\nabla| \stackrel{h}{h}\right|^{2}\right|^{2}=4|\stackrel{h}{h}|^{2}|\nabla| \stackrel{\circ}{h}||^{2}$. From the definition of $f_{\varepsilon}$ we obtain

$$
-\frac{1}{2} \int_{M} \Delta|\stackrel{\circ}{h}|^{2}|\stackrel{\circ}{h}|^{2} f_{\varepsilon}^{-4}=-2 \int_{\Omega_{\varepsilon}}|\nabla| \stackrel{\circ}{h}||^{2}|\stackrel{\circ}{h}|^{-2}+\left.\frac{2}{\varepsilon^{4}} \int_{\Omega_{\varepsilon}^{c}}|\nabla| \stackrel{\circ}{h}\right|^{2}|\stackrel{\circ}{h}|^{2} .
$$

Moreover the definition of $f_{\varepsilon}$ implies

$$
\left.2 \int_{M}|\nabla| \stackrel{h}{h}\right|^{2}|\stackrel{\circ}{h}|^{2} f_{\varepsilon}^{-4}=\left.2 \int_{\Omega_{\varepsilon}}|\nabla| \stackrel{\circ}{h}\right|^{2}|\stackrel{\circ}{h}|^{-2}+\frac{2}{\varepsilon^{4}} \int_{\Omega_{\varepsilon}^{c}}|\nabla| \stackrel{\circ}{h}||^{2}|\stackrel{h}{h}|^{2}
$$

and by integrating by parts, using the definition of $f_{\varepsilon}$ and taking into account that $\nabla f_{\varepsilon}=0$ in $\Omega_{\varepsilon}^{c}$ we get

$$
\begin{aligned}
\int_{M} \nabla_{i} \nabla_{j} H \stackrel{\circ}{h}_{i j}|\stackrel{\circ}{h}|^{2} f_{\varepsilon}^{-4}= & -\frac{1}{2} \int_{M}|\nabla H|^{2}|\stackrel{\circ}{h}|^{2} f_{\varepsilon}^{-4}-\frac{1}{\varepsilon^{4}} \int_{\Omega_{\varepsilon}^{c}} \stackrel{\circ}{h}_{i j} \nabla_{i}|\stackrel{\circ}{h}|^{2} \nabla_{j} H \\
& +\int_{\Omega_{\varepsilon}} \stackrel{\circ}{h}_{i j} \nabla_{j} H \nabla_{i}|\stackrel{\circ}{h}|^{2}|\stackrel{\circ}{h}|^{-4} .
\end{aligned}
$$

So from $(13),(14),(15),(16)$ and from the definition of $f_{\varepsilon}$ we obtain

$$
0=\int_{\Omega_{\varepsilon}} R+\frac{1}{\varepsilon^{4}} \int_{\Omega_{\varepsilon}^{c}} R|\stackrel{\circ}{h}|^{4}-\frac{2}{\varepsilon^{4}} \int_{\Omega_{\varepsilon}^{c}} \stackrel{\circ}{h}_{i j} \nabla_{i}|\stackrel{\circ}{h}|^{2} \nabla_{j} H+\left.\frac{4}{\varepsilon^{4}} \int_{\Omega_{\varepsilon}^{c}}|\nabla| \stackrel{\circ}{h}\right|^{2}|\stackrel{\circ}{h}|^{2} .
$$

From Gauss-Bonnet Theorem we get

$$
0=4 \pi \chi(M)-\int_{\Omega_{\varepsilon}^{c}} R-\frac{2}{\varepsilon^{4}} \int_{\Omega_{\varepsilon}^{c}} \stackrel{\circ}{h}_{i j} \nabla_{i}|\stackrel{\circ}{h}|^{2} \nabla_{j} H+\left.\frac{4}{\varepsilon^{4}} \int_{\Omega_{\varepsilon}^{c}}|\nabla| \stackrel{\circ}{h}\right|^{2}|\stackrel{\circ}{h}|^{2}+\frac{1}{\varepsilon^{4}} \int_{\Omega_{\varepsilon}^{c}} R|\stackrel{\circ}{h}|^{4} .
$$


Moreover, tracing Gauss equation (5) we get $R=2 c+H^{2}-|h|^{2}$, which in terms of $h$ becomes $R=\frac{1}{2} H^{2}-|\stackrel{\circ}{h}|^{2}+2 c ;$ so (17) yields

$$
\begin{aligned}
0= & 4 \pi \chi(M)-\int_{\Omega_{\varepsilon}^{c}}\left(\frac{1}{2} H^{2}-|\stackrel{\circ}{h}|^{2}+2 c\right)-\frac{2}{\varepsilon^{4}} \int_{\Omega_{\varepsilon}^{c}} \stackrel{\circ}{h}_{i j} \nabla_{i}|\stackrel{\circ}{h}|^{2} \nabla_{j} H \\
& +\left.\frac{4}{\varepsilon^{4}} \int_{\Omega_{\varepsilon}^{c}}|\nabla| \stackrel{\circ}{h}\right|^{2}|\stackrel{\circ}{h}|^{2}+\frac{1}{\varepsilon^{4}} \int_{\Omega_{\varepsilon}^{c}}\left(\frac{1}{2} H^{2}-|\stackrel{\circ}{h}|^{2}+2 c\right)|\stackrel{\circ}{h}|^{4} .
\end{aligned}
$$

From (12), we obtain

$$
\begin{aligned}
& 0=4 \pi \chi(M)+\frac{2}{\varepsilon^{4}} \int_{\Omega_{\varepsilon}^{c}}|\nabla \stackrel{\circ}{h}|^{2}|\stackrel{h}{h}|^{2}-\frac{1}{\varepsilon^{4}} \int_{\Omega_{\varepsilon}^{c}}|\nabla H|^{2}|\stackrel{h}{h}|^{2} \\
& -\int_{\Omega_{\varepsilon}^{c}}\left(\frac{1}{2} H^{2}-|\stackrel{h}{h}|^{2}+2 c\right)+\frac{1}{\varepsilon^{4}} \int_{\Omega_{\varepsilon}^{c}}\left(\frac{1}{2} H^{2}-|\stackrel{\circ}{h}|^{2}+2 c\right)|\stackrel{\circ}{h}|^{4}
\end{aligned}
$$

i.e.

$$
0 \geq 4 \pi \chi(M)+\frac{2}{\varepsilon^{4}} \int_{\Omega_{\varepsilon}^{c}}|\nabla \stackrel{\circ}{h}|^{2}|\stackrel{h}{h}|^{2}-\frac{1}{\varepsilon^{4}} \int_{\Omega_{\varepsilon}^{c}}|\nabla H|^{2}|\stackrel{\circ}{h}|^{2}-C \operatorname{Vol}\left(\Omega_{\varepsilon}^{c}\right),
$$

which is (1), where $C$ is given by

$$
C=\frac{1}{2}\|H\|_{\infty}^{2}+4|c|+1
$$

if $\varepsilon \leq 1$. Now since $\left|\Omega_{\varepsilon}^{c}\right| \rightarrow\left|\Omega_{0}^{c}\right|$ as $\varepsilon \rightarrow 0$, then, if (2) holds, then $\operatorname{Vol}\left(\Omega_{0}^{c}\right)>0$. This concludes the proof of Theorem 1.1 .

Proof of Corollary 1.2. The proof of Corollary 1.2 is an immediate application of Theorem 1.1 for immersed spheres (i.e. $\chi(M)=2$ ), since each of the three conditions implies inequality $(2)$.

Acknowledgments. G.C and A.R. have been partially supported by the "Gruppo Nazionale per l'Analisi Matematica, la Probabilit e le loro Applicazioni" (GNAMPA) of the "Istituto Nazionale di Alta Matematica" (INdAM). L.V. has been partially supported by the G.N.S.A.G.A. of I.N.d.A.M. This manuscript was written while G.C. and A.R. were visiting the Department of Mathematics of the University of Turin, which is acknowledged for the hospitality. 


\section{REFERENCES}

[1] H. Alencar and M. do Carmo, Hypersurfaces with constant mean curvature in spheres, Proc. Amer. Math. Soc. 120 (1994), 1223-1229.

[2] B. Andrews, H. Li, Embedded constant mean curvature tori in the three-sphere, J. Diff. Geom. 992 (2012), 169-189.

[3] S. Brendle, Embedded minimal tori in $\mathbb{S}^{3}$ and the Lawson conjecture, Acta Mathematica 211 (2013), 177-190.

[4] S. Bochner, Vector fields and Ricci curvature, Bull. Amer. Math. Soc. 52 (1946), 776-797.

[5] J.-P. Bourguignon, The magic of Weitzenböck formulas, Variational Methods Paris (1998). Progess in Nonlinear Differential Equations and Applications IV, Birkauser, 1990, pp. 251-271.

[6] G. Catino, A remark on compact hypersurfaces with constant mean curvature in space forms, Bull. Sci. Math. 1408 (2016), 901-907.

[7] G. Catino, On conformally flat manifolds with constant positive scalar curvature, Proc. Amer. Math. Soc. 144 (2016), 2627-2634.

[8] S.S. Chern, On surfaces of constant mean curvature in a three-dimensional space of constant curvature. Lecture notes in Math. 1007 (1983), 104-108.

[9] S.S. Chern, M. do Carmo, S. Kobayashi, Minimal submanifolds of a sphere with second fundamental form of constant length Functional Analysis and Related Fields (1970), 59-75.

[10] C. De Lellis, S. Müller, Optimal rigidity estimates for nearly umbilical surfaces, J. Diff. Geom. 69 (2005) 75-110.

[11] M. do Carmo, Riemannian geometry. Mathematics: Theory and Applications, vol. 290, Birkhäuser, 1992.

[12] H. Hopf, Über Flächen mit einer Relation zwischen den Hauptkrümmungen, Math. Nachr. 4 (1951), 232249.

[13] W. H. Meeks, P. Mira, J. Perez, A. Ros, Constant mean curvature spheres in homogeneous three-manifolds, arXiv: 1706.09394.

[14] K. Nomizu, B. Smyth, A formula of Simons' type and hypersurfaces with constant mean curvature, J. Diff. Geom. 3 (1969), 367-377.

[15] J. Simons, Minimal varieties in Riemannian manifolds, Ann. of Math. 882 (1968), 62-105.

[16] K. Smoczyk, Evolution of spacelike surfaces in $A d S_{3}$ by their Lagrangian angle, Math. Ann. 355 (2013), 14431468.

[17] H.C. Wente. Counterexample to a conjecture of H. Hopf, Pacific. J. Math. 121 (1986), 193-243.

[18] S.T. Yau. Submanifolds with constant mean curvature. I, II, Amer. J. Math. 96 (1974), 346-366.

G. Catino, Dipartimento di Matematica, Politecnico di Milano, Piazza Leonardo da Vinci 32 , 20133 Milano, Italy.

E-mail address: giovanni.catino@polimi.it

A. Roncoroni, Dipartimento di Matematica F. Casorati, Università di Pavia, Via Ferrata 5, 27100 Pavia, Italy.

E-mail address: alberto.roncoroni01@universitadipavia.it

L. Vezzoni, Dipartimento di Matematica G. Peano, Università di Torino, Via Carlo Alberto 10, 10123 TORINo, ITALy.

E-mail address: luigi.vezzoni@unito.it 